\title{
Paper Maps: Improving the Readability of Scientific Papers via Concept Maps
}

\author{
Lorenzo Amabili* \\ University of Groningen \\ Nicole Sultanum ${ }^{\dagger}$
University of Toronto
}

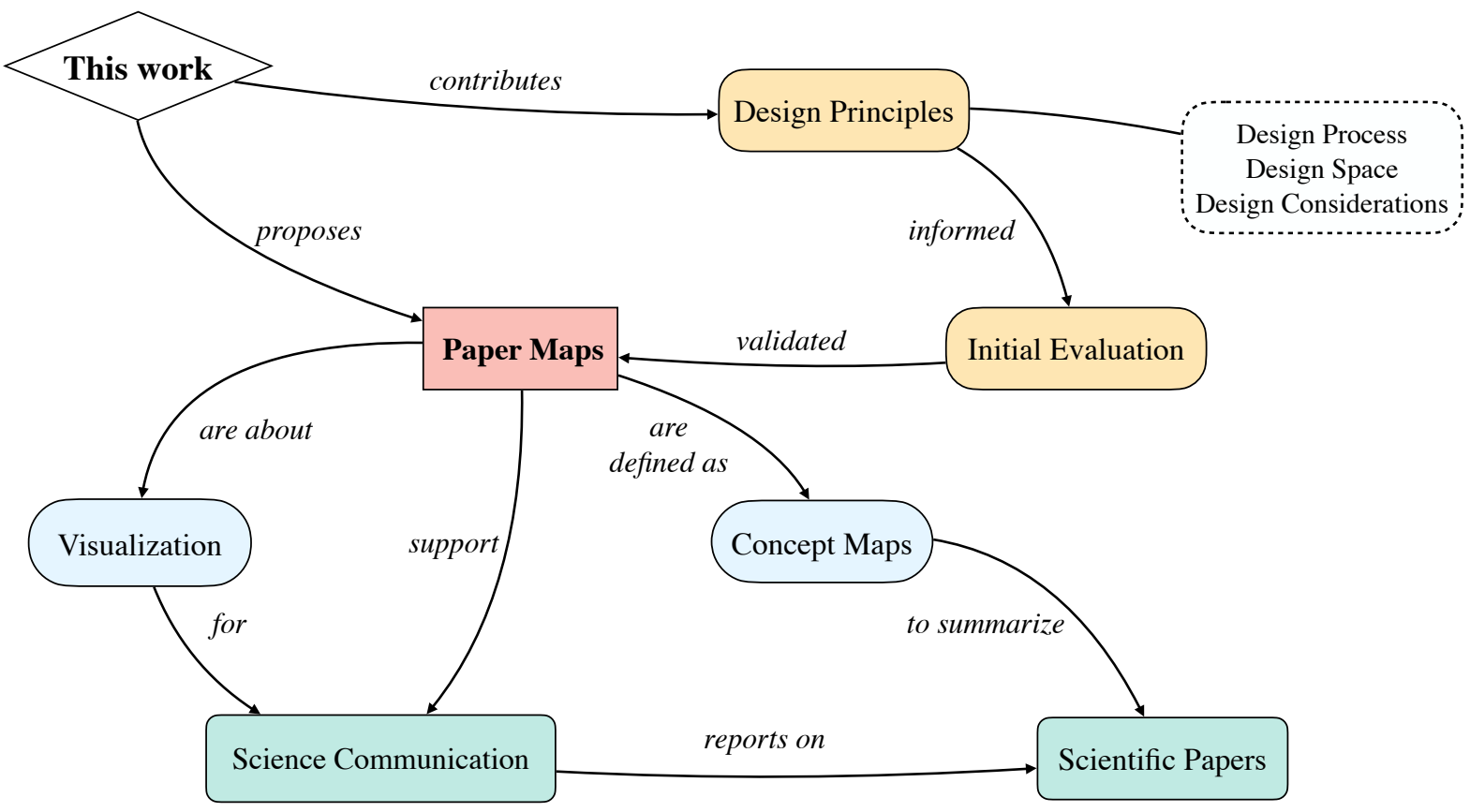

Figure 1: Paper map of this paper. Each node represents a concept and each link represents the relation between concepts extracted from the document. The visual encoding provides further information about each node such as the specificity of concepts (i.e., node border radius) and type of concepts (i.e., node color); closely related subconcepts are enclosed in node boxes with dotted border and linking words are encoded in italics.

\begin{abstract}
Given the wealth of scientific publications, perusing papers is becoming a larger and more complex burden, especially for junior researchers. In this work, we suggest a visualization-based method to mitigate this problem via the use of paper maps, i.e., concept maps for the summarization of scientific papers. We provide design principles of paper maps and discuss design considerations based on exploratory design studies. We also conducted an initial evaluation for assessing the effectiveness of paper maps in summarizing scientific papers, suggesting that paper maps can improve the readability of scientific papers.
\end{abstract}

Index Terms: Human-centered computing-VisualizationVisualization techniques-Graph drawings; Human-centered computing - Visualization-Empirical studies in visualization

\section{INTRODUCTION}

Scientific papers are at the base of science communication. Researchers must read a significant number of papers to keep up with

*e-mail: 1.amabili@rug.nl

†e-mail: nicolebs@cs.toronto.edu the state-of-the-art in their fields. Considering the large amount of scientific papers available nowadays, triaging and reading papers can be complex and time-consuming, especially for junior researchers. Paper abstracts play an important role in alleviating this issue and supporting paper selection and reading, but still require dedicated time and attention. Recently, visual abstracts have been suggested to provide a visual summary of the information presented in an abstract [16] as a first glimpse of a scientific paper, to make research works as accessible as possible without compromising the quality of the scientific content.

In this work, we take this concept a step further and explore the notion of paper maps, concept maps for paper summarization. Concept maps are node-link diagrams where concepts are interconnected [15], showing relations among related concepts to schematize a main object of study and visually convey structural understanding [5]. Similarly, paper maps aim to summarize scientific paper content and can be used to guide researchers throughout paper reading. As the concepts presented in scientific papers can be difficult for nonexperts to process, we posit that paper maps may facilitate access to a high-level understanding of the material presented. Therefore, we invite authors to use paper maps for providing readers with an additional resource for understanding the content of their papers [4]. We outline design principles and propose a design space for paper maps, specifically applied to representing academic contributions. We showcase the design space with some paper maps of articles within the 6 VIS areas [25] and we provide preliminary design 
recommendations to support authors of scientific papers to compose visual summaries of their papers. We also conducted an initial evaluation for collecting feedback about the effectiveness of paper maps in facilitating reading papers and discuss the results emerging from this study together with their implications.

\section{Related Work}

Previous studies about concept maps and alternative representations for scientific knowledge motivated our work which we discuss in this section.

\subsection{Concept Maps}

Concept maps are "node-link diagrams in which each node represents a concept and each link identifies the relationship between the two concepts it connects" [21]. They are also defined as "graphical tools for organizing and representing knowledge" [15]. In psychology, hierarchically structured concept maps are referred as "cognitive maps" [7], which denote explicit and structured representations of knowledge or understanding [11]. Initially created as a tool to represent children's learning and knowledge of science, concepts maps have also been found useful as a teaching tool, to "promote meaningful learning" $[4,5,15]$ and as an evaluation tool, allowing instructors to "provide feedback to students" and to "assess learning" [4, 15]. Moreover, a meta analysis by Schroeder et al. [21] found that concept maps have a positive effect on learning, with a higher effect size for constructing maps, but still positive for reading maps for educational purposes.

To create concept maps, Novak and Cañas [15] outlined a general process that starts with constructing a main focus question of the concept map, identifying the key concepts applying to the related domain for which "usually 15 to 25 concepts will suffice", then constructing a preliminary concept map by placing concepts around, and finally adding links between concepts to help illustrate how concepts are related to one another. This process can be time consuming, and other works, such the work of Falke and Gurevych [9], propose a method to automatically extract concept maps for document summarization, allowing for document collections to be more easily explored.

The above works provided us with a theoretical background on concept maps. Although they have been deeply studied, no studies investigated the use of concept maps specifically to support the visual representation of academic knowledge. This motivated us to explore the application of concept maps in science communication.

\subsection{Representing Scientific Knowledge}

A number of research works proposed ideas for alternative representation of academic publications, several of them visual. Ramos and Concepcion [16] claim that an illustration representing the content of paper abstracts can improve the communication of scientific knowledge and they suggested the use of visual abstracts, i.e., some form of illustrations aimed at providing a quick overview of scientific papers. Wang et al. [26] proposed the use of data comics to summarize and report outcomes of controlled studies in a more compelling and understandable manner, and outline basic communication principles to author them. Dragicevic et al. [6] proposed an interactive embodiment for academic papers based on explorable multiverse analysis that allows for alternative analysis pathways to be seamlessly integrated into textual and visual components of the paper. Another solution was proposed by Riede et al. [17] who developed the full-metadata format, a scientific format for text-based data files, which facilitates storing and communicating tabular data sets. On the whole, several of the above alternative representations for scientific knowledge have been suggested to improve the accessibility and transparency of scientific papers. Following this trend, we envision that scientific papers including a paper map may provide a more intuitive and concise representation to schematize the document.

\section{PAPER MAPS}

Although concept maps are well-known, the notion of paper maps should be further outlined. In this section, we explain what makes a concept map a paper map and why we consider paper maps as a promising solution for improving the accessibility of scientific papers. We also illustrate how to create paper maps for summarizing scientific paper and discuss initial design considerations.

\subsection{Defining Paper Maps}

A paper map is a concept map with a specific focus question: "what is this research work about?". While concept maps can be used for different purposes such as organizing knowledge and enhancing learning $[4,5,15]$, paper maps are (a) primarily created by authors and (b) specifically employed for improving research communication by summarizing scientific papers. Paper maps represent only the paper content and should cover key concepts and vocabulary used in the scientific paper, in order to facilitate the subsequent reading of the entire document [24]. We illustrate an example of a paper map for this very publication in Fig. 1.

Paper maps are concise as they are high-level representations of scientific knowledge aiming to expedite understanding. This means that clarity takes precedence over subjectivity and details, and that the number of concepts should be kept minimal.

Paper maps are self-contained. They are mini-documents of their own as they are detached from the related papers: a paper map can be read without having the scientific paper available and it still provides the reader with an overview of the paper.

Paper maps are visual stories. They can be considered as fastforward stories of the related papers. To improve the readability of the map and the memorability of the concepts illustrated, paper maps present storytelling elements such as characters (i.e., the concepts) and a plot (i.e., the map flow).

\subsection{Design Process}

The process of creating paper maps is aimed at answering the question of "what is this research work about?" and consists of a sequence of steps with repeated refinement. We propose steps to author paper maps building based on a previous analysis of the design process of concept maps from the literature [24] as follows:

(S1) Identify relevant concepts. The first step for designing a paper map is to identify the key concepts that are most descriptive of the work, preferably using words from the document itself

(S2) Rank the concepts by specificity. The identified concepts are ranked from the most general to the most specific, according to their use in the document.

(S3) Locate concepts in the map. Starting from the most general ones, the concepts are placed in a tentative layout.

(S4) Relate the concepts with linking words. Words expressing the concept relationships across different concepts are identified to connect the different concepts.

(S5) Repeat steps (S1-S4) until sufficient summary. This process of identification and placement of relevant concepts and their relations is repeated until the concepts and links are found representative enough of the scientific paper and the resulting scope, topology, and layout form a concise self-contained story.

(S6) Visually encode the information related to the concepts and relations. Information associated to each concept (e.g., concept type or specificity) is visually encoded into concept 


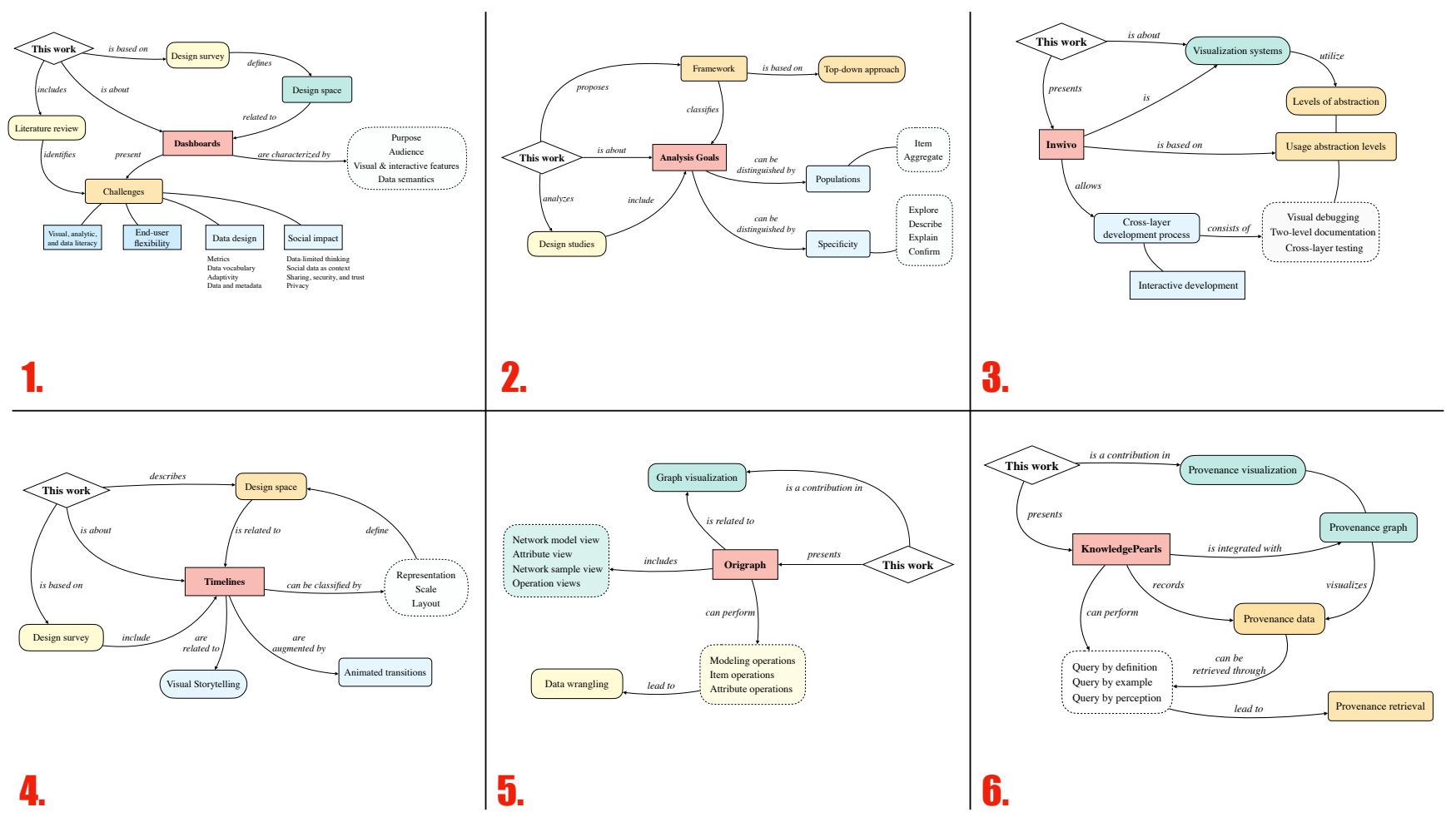

Figure 2: Exploration of the paper maps design space. Each frame shows a paper map related to a scientific paper of one of the 6 VIS areas [25], created to explore the paper maps design space (1. [20], 2. [12], 3. [10], 4. [3], 5. [2], and 6. [22]). The concepts are categorized per type (node color) and specificity (node border radius); closely related subconcepts are enclosed in node boxes with a dotted border and linking words are encoded in italics.

blocks and links by making use of visual variables [18] to make the maps more expressive and intuitive to read.

(S7) Refine the paper map by repeating steps (S1-S6). The map design is refined several times to find the most appropriate paper map in the design space of paper maps.

\subsection{Design Space}

To explore the design space of paper maps, we created 6 paper maps of articles within each of the newly defined VIS areas [25] depicted in Fig. 2 (i.e., 1. [20], 2. [12], 3. [10], 4. [3], 5. [2], and 6. [22]). Based on the results of this exploratory study and on past works [15,21], we identified a design space of paper maps based on three dimensions, content, representation, and arrangement, which we define as follows:

(D1) Content. It regards how the concepts and their relations are selected, added and discarded which is driven by the paper content (i.e., data), the target audience (i.e., users) and the focus question(s) (i.e., tasks) [14] of the paper map.

(C1) Level of detail. It is about to the amount of information provided with a paper map (i.e., the number of concepts and relations included into the paper map). In Fig. 2, the paper maps have a low level of detail as the number of concepts and relations is small $(<15)$. An exception is the paper map in frame 1. Fig. 2 which has a high level of detail.

(C2) Level of abstraction. It regards the specificity of the concepts included into the paper map. In Fig. 2, we can observe that the paper map in frame 1. includes both general concepts such as Challenges and specific concepts such as End-user flexibility.

(D2) Representation. It is about how the concepts and their relations are visually represented which is based on the use of visual variables [18]. In Fig. 2, color hue, color value and shape are used to enrich the visual elements of the paper maps.

(R1) Nodes. They are the labelled visual elements used for representing the paper map concepts.

(R2) Links. They are the labelled visual elements used for representing the relations between paper map concepts.

(D3) Arrangement. It regards how the concepts and their relations are organized and deployed in the space used for displaying the paper map.

(A1) Structure. It is the topology of the paper map which determines how the concepts are linked together. The structure of the paper map can also be hierarchical. This is the case for the paper map in frame 1. of Fig. 2 where the concept Literature review is directly linked to the starting node This work as it is a related concept whereas the less related concept End-user flexibility is not. In contrast, the paper map in frame 4. of Fig. 2 has a non-hierarchical structure.

(A2) Layout. It is how the concept nodes and links are deployed in the space dedicated to the paper map. For instance, in frame 4. of Fig. 2 the paper map layout is radial as the key word Timelines is at the center of the map where the subordinate concepts are all around it. 
Differently, in frame 2. of Fig. 2 the paper map layout is horizontal.

(A3) Orientation. It is how the paper map is directed and it determines how it should be read. In Fig. 2, paper maps have a top-bottom, left-right orientation as the starting node This work is always located on the top or on the left-hand side of the map. However, the paper map in frame 5. of Fig. 2 is an exception as the starting node is located on the right-hand side of the map.

\subsection{Design Considerations}

During our exploratory design studies, we realized that several non-obvious design choices such as the number of concepts and relations to include in the paper map must be taken. For this reason, we provide paper authors with general guidelines to follow when creating and including a paper map to their papers, based on our observations and direct experience of creating different paper maps designs assessed and refined through the do-it-yourself and pair analytics evaluation techniques [8].

Paper maps should have a top-bottom, left-right orientation. We are used to read papers, texts, and visualizations from top to bottom and from left to right; so we do with paper maps [15]. Maintaining similar orientations while designing paper maps is expected to improve the map readability. We suggest to locate a starting concept at the top or left-hand side of the map and a concluding concept at the bottom or right-hand side of the map. We do acknowledge this design recommendation may not hold in all cases, as cultural expectations must also be taken into account when designing paper maps for different audiences.

Avoid dense paper maps. Independently of the level of detail adopted, paper maps should be easy to read and navigate. According to our exploratory studies, balanced paper maps in terms of visual elements deployment seem to be perceived more readable. Discarding the redundant concepts together with spacing and spreading out the remaining concepts for covering the entire area reserved to the paper map can improve map readability.

Consider Gestalt principles. The design of paper maps can be improved by following Gestalt principles [19], in particular proximity, similarity, symmetry and continuation. Therefore, we suggest to locate similar elements close together and to visually encode their similarity, for example by using the same color hue to fill the node boxes of similar concepts. Making similar elements symmetric also reinforces their relation and eases the map reading. Furthermore, nodes and linking words are perceived more related if aligned on a line or around a curve.

There is not one good paper map design for a given paper. We realized that paper maps depend on several factors such as individual and cultural differences. Although it is possible to narrow the design space down by following specific criteria (e.g., the design recommendations provided in this paper), there does not exist a corresponding ubiquitous paper map for a given paper. Similarly as writing a paper abstract, creating a paper map encompasses selecting one design solution among a large solution space which also achieve the objective of effectively summarizing scientific papers.

\section{INITIAL FEEDBACK}

We conducted an initial evaluation on the effectiveness of paper maps in summarizing scientific papers. To this end, each of the authors of this work created a paper map related to one of our previous works $[1,23]$, shown in Fig. 3 and Fig. 4, respectively. Following, we asked 5 visualization researchers ( $3 \mathrm{MSc}$ students, $1 \mathrm{PhD}$ student and 1 professor) to (a) read the two given paper maps and answers
5 questions about each paper map, and then (b) answer 6 questions about paper maps in general.

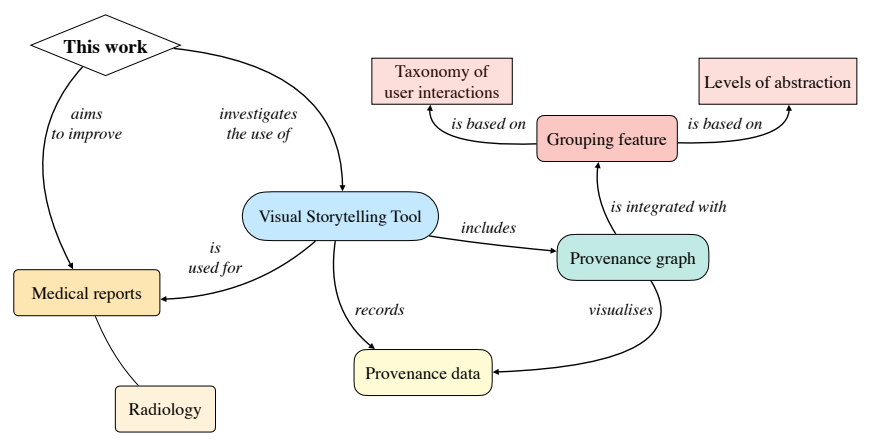

Figure 3: First paper map of one of our previous works [1] which was shown to the study participants. In this map, the node color is encoding the type of concept and the node border radius is encoding the specificity of the concept. The linking words are encoded in italics.

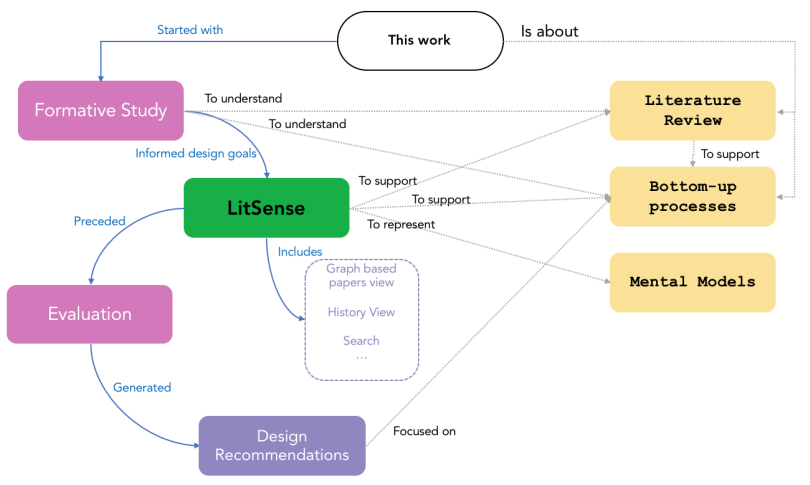

Figure 4: Second paper map of one of our previous works [23] which was shown to the study participants. In this map, both space and color encode the type of concept. The left nodes showcases research steps, and the right nodes list keyword concepts and how they associate to each step. Closely related subconcepts are enclosed in a node box with a dotted border.

\section{For each paper map:}

(Q1) What do you think is the main contribution of the work depicted in this paper map?

(Q2) How would you summarize the content of the paper depicted by this paper map?

(Q3) Do you feel you are able to assess from the paper map if this work is relevant to your research interests, or not? Why?

(Q4) What about this paper map is helpful/not helpful to you to have an overview of the related paper?

(Q5) Is the paper map easy/hard to read? How?

\section{Overall reflections:}

(Q6) Would you like/not like to have paper maps like these at the beginning of scientific papers? Why?

(Q7) What would you change/improve in these paper maps to make them more effective in summarizing scientific papers? 
(Q8) How/When would you see yourself using such paper maps? Before reading the abstract? Recall a paper you've read? Other?

(Q9) How does this compare to written abstracts, in your opinion?

(Q10) Did you go over the papers to answer the questions, and how much did you cover from each?

\section{(Q11) Any other thoughts not covered by the previous questions?}

Overall feedback was positive and consistent across researchers. All participants were able to correctly identify the main contribution and summarize the content of the scientific paper depicted by the paper maps (Q1, Q2). All of them found they were able to assess from the paper map if the associated work was relevant to their research interests or not (Q3). All participants also found value in having paper maps like those presented at the beginning of scientific papers to have an overview of the content and structure of scientific papers (Q4).

Moreover, the following quotes helped us ascertain that paper maps facilitate the reading process of scientific papers, recalling their content and triaging papers: "I would probably look at the paper map before reading the abstract. If it includes elements that are relevant to me I would then examine their connections in the map and based on this go towards reading the abstract. For quickly recalling the main elements of the paper it also seems useful as it seems to take less mental power to look at the map than read the abstract."; and "I would use it before I have read the abstract and to use it as a general selection criteria to select which papers are useful to me. I think it is also important to see which section of the paper could be directly related to my research, allowing me to skip generic introductions or similar concepts across the same domain." On the other hand, some of the participants (3/5) suggested to improve the visual encoding and arrangement of the paper maps, which was not clear for all participants, although they confirmed that the current paper maps were helpful to provide an overview of the related paper (Q7). On this issue, one participant remarked that "the standardization of the visual encoding and of the arrangement" would make paper maps more effective in summarizing scientific papers. Similarly, another participant commented with "I would like a standardized model for all papers or else in order to compare research" to a related question (Q6). A third participant explicitly highlighted his concern about the time and skills creating paper maps would need by commenting with "It seems helpful to have paper maps at the beginning of scientific papers as the paper maps seem to give more insight to the contents of the paper than would ordinary keywords. However, I do not see myself quickly making a paper map for a submitted paper, but if it ever becomes required to do so in the future it would be beneficial to have some guidelines in how to create such a map." (Q8). Based on those comments, we expect that a standardization of the paper maps design would let paper authors to create effective paper maps more quickly and it must be addressed in future work.

\section{Discussion}

We envisioned that concise self-contained visual stories of papers via paper maps can help summarize scientific contributions, which was confirmed by our preliminary study. Among the insights gained during the study, we observed that sparse paper maps (i.e., paper maps including spread out and spaced concepts) seem to be perceived as more readable. This is of particular interest as it is in contrast with previous research work on concept maps which "translates relationship judgments into distances so that highly related ideas are close together and less related ideas are further apart." [5]. However, further design studies are required to better understand the tradeoff between encoding the semantic similarity among concepts with the distance among concepts and spacing the concepts for entirely covering the paper map area to improve the map readability.

From our initial evaluation study of the value of paper maps with 5 researchers, we learned that all participants would like to use paper maps as they consider them helpful in providing an overview of the paper content and structure. This confirms an actual problem in science, namely that it still lacks effective tools for simplifying research practice and there is a need for strategies to make science more accessible and intuitive. In this regard, study participants found paper maps were easy to read, effective for recalling information related to the paper, and useful for skimming large amounts of papers. This encouraging insights motivates us to continue to investigate the use of paper maps and to define more specific design guidelines towards further standardization of paper maps, as suggested by some participants. However, standardizing the paper map design presents pros and cons. On one hand, it would improve the process of designing paper maps in terms of time required and would enhance the paper map design in terms of map readability and map content recalling. With fixed standards, paper map design would also become less ambiguous and prone to misunderstanding and favor reproducibility and transparency of the paper summarization. On the other hand, conventionality restricts authors' freedom in expressing a visual summary of their papers in their own terms. Different authors generate different paper maps which are all possibly meeting the design requirements. Therefore, individual differences in designing and perceiving visual representations can hinder converging into a unique paper map design. A possible solution to this dichotomy is to define precise and specific guidelines which further narrow the design space of paper maps down, but without requiring a fixed design. In this way, the paper map design would follow design criteria established in advance, but still allowing some variations.

An extensive study on how to achieve this can consist in observing a significant number of potential paper maps authors creating paper maps for their papers. This process would generate enough data for outlining guidelines based on common ground across different paper maps to define design standards and characterize design challenges. Moreover, paper maps authors can also be interviewed a second time for feedback on previously created paper maps to further refine and validate design choices. Finally, a group of potential paper maps readers can be asked to evaluate the effectiveness of paper maps and to compare them with generic visual abstracts to validate our approach. This study would also lead to the creation of paper map templates to ease the paper map design process. This is extremely relevant as we learned from our preliminary study that some authors may not feel comfortable in creating paper maps themselves, and creating effective paper maps can be non-trivial and requiring additional time. Although we believe that, in the era of open science and in a society by now used to visual content, adding paper maps into scientific papers can be worth the time they need to be created and this initial effort of becoming familiar with the paper map creation can pay back in the long term, in future, specific tools for automatically generating paper maps can also be developed by building upon current related tools [13].

At this stage of our work, we limited ourselves to propose a visualization method for improving science communication, to provide general guidelines resulting from an initial design study, and to invite VIS researchers to consider adding paper maps into their papers. We envision that paper maps could outweigh generic visual abstracts in the long run. Due to their standardized and modular nature, readers can associate, compare and link paper maps of different papers to build new knowledge through the creation of schemas and mental models representing this knowledge. We conceived paper maps as supplementary tools of scientific papers facilitating reading tasks such as skimming and triaging papers which can be stored, browsed and read from ad-hoc libraries. Together with adding paper maps as teasers on top of the abstract of their papers, paper authors could 
also make paper maps available as external files for allowing their collection and access. This would imply that the process of creating literature reviews can be sped up, fostering an increase in the number of scientific reviews which would summarize extended research areas, promote more informed interdisciplinary studies, and ease formulating research questions and identifying gaps and open challenges for nourishing future investigations.

\section{Conclusion}

In this work, we propose the notion of paper maps, i.e., concept maps for summarization of scientific papers that are created by authors and included with the publication. We conducted exploratory studies to identify the design space of paper maps and to define the process steps for designing paper maps. Based on that, we provided initial design recommendations for helping paper authors to create paper maps for their papers. Through an initial evaluation, we found paper maps are useful to have an overview of the paper, to skim scientific papers, and to easily recall paper content. Most importantly, study participants explicitly expressed that they would like to have paper maps in scientific papers. However, they would also like to obtain a standardization of the paper map design. This motivates us in continuing investigating the use of paper maps for improving science communication and to extend this study in the future. A follow-up study could define specific guidelines and standards for designing paper maps through focus group interviews in which researchers create paper maps and discuss their design choices.

\section{REFERENCES}

[1] L. Amabili, J. Kosinka, M. A. J. v. Meersbergen, P. M. A. v. Ooijen, J. B T. M. Roerdink, P. Svetachov, and L. Yu. Improving provenance data interaction for visual storytelling in medical imaging data exploration. In EuroVis 2018 - Short Papers. The Eurographics Association, 2018. doi: 10.2312/eurovisshort.20181076

[2] A. Bigelow, C. Nobre, M. D. Meyer, and A. Lex. Origraph: Interactive network wrangling. CoRR, abs/1812.06337, 2018.

[3] M. Brehmer, B. Lee, B. Bach, N. H. Riche, and T. Munzner. Timelines revisited: A design space and considerations for expressive storytelling. IEEE Transactions on Visualization and Computer Graphics, 23(9):2151-2164, 2017. doi: 10.1109/TVCG.2016.2614803

[4] B. J. Daley and D. M. Torre. Concept maps in medical education: an analytical literature review. Medical Education, 44(5):440-448, 2010. doi: 10.1111/j.1365-2923.2010.03628.x

[5] G. M. Diekhoff and K. B. Diekhoff. Cognitive maps as a tool in communicating structural knowledge. Educational Technology archive, 22:28-30, 1982.

[6] P. Dragicevic, Y. Jansen, A. Sarma, M. Kay, and F. Chevalier. Increasing the transparency of research papers with explorable multiverse analyses. Proceedings of the 2019 CHI Conference on Human Factors in Computing Systems, 2019. doi: 10.1145/3290605.3300295

[7] C. Eden. Cognitive mapping. European Journal of Operational Research, 36(1):1-13, 1988. doi: 10.1016/0377-2217(88)90002-1

[8] N. Elmqvist and J. S. Yi. Patterns for visualization evaluation. In Proceedings of the 2012 BELIV Workshop: Beyond Time and Errors Novel Evaluation Methods for Visualization, BELIV '12. Association for Computing Machinery, New York, NY, USA, 2012. doi: 10.1145/ 2442576.2442588

[9] T. Falke and I. Gurevych. Graphdocexplore: A framework for the experimental comparison of graph-based document exploration tech niques. In Proceedings of the 2017 Conference on Empirical Methods in Natural Language Processing: System Demonstrations, pp. 19-24, 2017. doi: 10.18653/v1/D17-2004

[10] D. Jönsson, P. Steneteg, E. Sundén, R. Englund, S. Kottravel, M. Falk, A. Ynnerman, I. Hotz, and T. Ropinski. Inviwo - a visualization system with usage abstraction levels. IEEE Transactions on Visualization and Computer Graphics, 26(11):3241-3254, 2020. doi: 10. 1109/TVCG.2019.2920639
[11] R. M. Kitchin. Cognitive maps: What are they and why study them? Journal of Environmental Psychology, 14(1):1-19, 1994. doi: 10.1016/ S0272-4944(05)80194-X

[12] H. Lam, M. Tory, and T. Munzner. Bridging from goals to tasks with design study analysis reports. IEEE Transactions on Visualization and Computer Graphics, 24(1):435-445, 2018. doi: 10.1109/TVCG.2017. 2744319

[13] Z. Lu, M. Fan, Y. Wang, J. Zhao, M. Annett, and D. Wigdor. Inkplanner: Supporting prewriting via intelligent visual diagramming. IEEE Transactions on Visualization and Computer Graphics, 25(1):277-287, 2019. doi: 10.1109/TVCG.2018.2864887

[14] S. Miksch and W. Aigner. A matter of time: Applying a data - users tasks design triangle to visual analytics of time-oriented data. Computers Graphics, Special Section on Visual Analytics, 38:286-290, 2013. doi: $10.1016 /$ j.cag. 2013

[15] J. D. Novak and A. J. Cañas. The theory underlying concept maps and how to construct them. Florida Institute for Human and Machine Cognition, 1, 2006. doi: 10.12691/education-3-8-10

[16] E. Ramos and B. P. Concepcion. Visual abstracts: Redesigning the landscape of research dissemination. Seminars in Nephrology, 40(3):291297, 2020. Nephrology and Social Media. doi: 10.1016/j.semnephrol. 2020.04.008

[17] M. Riede, R. Schueppel, K. O. Sylvester-Hvid, M. Kühne, M. C. Röttger, K. Zimmermann, and A. W. Liehr. On the communication of scientific data: The full-metadata format. Computer Physics Communications, 181(3):651-662, Mar 2010. doi: 10.1016/j.cpc.2009.11. 014

[18] R. E. Roth. Visual variables. The International Encyclopedia of Geography, Wiley, 2016. doi: 10.1002/9781118786352.wbieg0761

[19] A. Rusu, A. J. Fabian, R. Jianu, and A. Rusu. Using the gestalt principle of closure to alleviate the edge crossing problem in graph drawings. In 2011 15th International Conference on Information Visualisation, pp. 488-493, 2011. doi: 10.1109/IV.2011.63

[20] A. Sarikaya, M. Correll, L. Bartram, M. Tory, and D. Fisher. What do we talk about when we talk about dashboards? IEEE Transactions on Visualization and Computer Graphics, 25(1):682-692, 2019. doi: 10. 1109/TVCG.2018.2864903

[21] N. L. Schroeder, J. Nesbit, C. J. Anguiano, and O. O. Adesope. Studying and constructing concept maps: a meta-analysis. Educational Psychology Review, 30:431-455, 2018. doi: 10.1007/s10648-017-9403 $-9$

[22] H. Stitz, S. Gratzl, H. Piringer, T. Zichner, and M. Streit. Knowledgepearls: Provenance-based visualization retrieval. IEEE Transactions on Visualization and Computer Graphics (VAST '18), 25(1):120-130, 2018. doi: 10.1109/TVCG.2018.2865024

[23] N. Sultanum, C. Murad, and D. Wigdor. Understanding and supporting academic literature review workflows with litsense. In Proceedings of the International Conference on Advanced Visual Interfaces, AVI '20. Association for Computing Machinery, New York, NY, USA, 2020. doi: $10.1145 / 3399715.3399830$

[24] J. J. Villalón and R. Calvo. Concept maps as cognitive visualizations of writing assignments. J. Educ. Technol. Soc., 14:16-27, 2011.

[25] VIS'21. The VIS area model for 2021+, 2021.

[26] Z. Wang, J. Ritchie, J. Zhou, F. Chevalier, and B. Bach. Data comics for reporting controlled user studies in human-computer interaction. IEEE Transactions on Visualization and Computer Graphics, 27(2):967-977, 2021. doi: 10.1109/TVCG.2020.3030433 